\title{
Protracted Respiratory Findings in Children Post-COVID-19 Infection
}

\author{
Shoshana Leftin Dobkin ${ }^{1}$, Joseph Collaco ${ }^{2}$, and Sharon McGrath-Morrow ${ }^{3}$ \\ ${ }^{1}$ The Children's Hospital of Philadelphia \\ ${ }^{2}$ Johns Hopkins Medical Institutions \\ ${ }^{3}$ Johns Hopkins Medical Insitutions
}

July 1, 2021

\begin{abstract}
Introduction: Although prolonged respiratory symptoms following SARS-CoV-2 infection have been reported in adults, there is a paucity of literature describing post-acute symptoms in pediatric patients following COVID-19. In this study we describe health data and respiratory findings in pediatric patients presenting with complaints of persistent respiratory symptoms following acute COVID-19 infection. Methods: This study included patients referred to Pulmonary Clinic at the Children's Hospital of Philadelphia between December 2020 and April $2021(\mathrm{n}=29)$. Inclusion criteria included a history of SARS-CoV-2 RNA positivity or confirmed close household contact. A retrospective chart review was performed and demographic, clinical, imaging, and functional test data were collected. Results: The mean age at presentation to clinic was 13.1 years (range: 4-19 years). Patients had persistent respiratory symptoms ranging from 1.3 to 6.7 months post-acute infection. Persistent dyspnea and/or exertional dyspnea were present in nearly all $(96.6 \%)$ of the patients at the time of clinic presentation. Other reported chronic symptoms included cough $(51.7 \%)$ and exercise intolerance $(48.3 \%)$. Fatigue was reported in $13.7 \%$ of subjects. Many subjects were overweight or obese $(62.1 \%)$ and eleven subjects had a prior history of asthma. Lung function was normal in most patients. The six-minute walk test (6MWT) revealed exercise intolerance and significant tachycardia in two-thirds of children tested. Conclusion: Exertional dyspnea, cough and exercise intolerance were the most common respiratory symptoms in children with post-acute COVID-19 respiratory symptoms seen in an outpatient pulmonary clinic. Lung function, however, was mostly normal, and exertional intolerance was frequently demonstrated using the 6MWT.
\end{abstract}

\section{Protracted Respiratory Findings in Children Post-COVID-19 Infection}

Shoshana C. Leftin Dobkin ${ }^{1}$, MD, Joseph M. Collaco ${ }^{2}$, MD, MS, PhD, Sharon A. McGrath-Morrow ${ }^{3}$, MD, MBA

${ }^{1}$ Division of Pulmonary Medicine, Children's Hospital of Philadelphia, Philadelphia, Pennsylvania, USA

${ }^{2}$ Eudowood Division of Pediatric Respiratory Sciences, Department of Pediatrics, Johns Hopkins Medical Institutions, Baltimore, Maryland, USA

${ }^{3}$ Division of Pulmonary Medicine, The Perelman School of Medicine at the University of Pennsylvania, Children's Hospital of Philadelphia, Philadelphia, Pennsylvania, USA

\section{Corresponding Author:}

Shoshana Leftin Dobkin, MD

Division of Pulmonary Medicine

Children's Hospital of Philadelphia 
Colket Translational Research Building

3501 Civic Center Boulevard, Philadelphia, PA 19104

Phone: (267) 854-5403

E-mail: leftindobs@chop.edu

Contributors: Conceptualization: all authors; Design: all authors; Data collection: SLD; Data analysis: SLD, JMC; Data interpretation: SLD, JMC, SAM; Manuscript writing: SLD; Manuscript review: all authors. SLD had full access to the study data and takes responsibility for the integrity of the data and the accuracy of the analysis.

Funding: The study was funded by The Children's Hospital of Philadelphia

Competing Interests: None

Keywords: exercise, functional capacity, breathlessness, pulmonary function, post-covid syndrome

Running Title: Long COVID in Children

Word Count: 1,754

\section{Abstract}

Introduction: Although prolonged respiratory symptoms following SARS-CoV-2 infection have been reported in adults, there is a paucity of literature describing post-acute symptoms in pediatric patients following COVID-19. In this study we describe health data and respiratory findings in pediatric patients presenting with complaints of persistent respiratory symptoms following acute COVID-19 infection.

Methods: This study included patients referred to Pulmonary Clinic at the Children's Hospital of Philadelphia between December 2020 and April 2021 ( $\mathrm{n}=29)$. Inclusion criteria included a history of SARS-CoV-2 RNA positivity or confirmed close household contact. A retrospective chart review was performed and demographic, clinical, imaging, and functional test data were collected.

Results: The mean age at presentation to clinic was 13.1 years (range: 4-19 years). Patients had persistent respiratory symptoms ranging from 1.3 to 6.7 months post-acute infection. Persistent dyspnea and/or exertional dyspnea were present in nearly all $(96.6 \%)$ of the patients at the time of clinic presentation. Other reported chronic symptoms included cough (51.7\%) and exercise intolerance (48.3\%). Fatigue was reported in $13.7 \%$ of subjects. Many subjects were overweight or obese $(62.1 \%)$ and eleven subjects had a prior history of asthma. Lung function was normal in most patients. The six-minute walk test (6MWT) revealed exercise intolerance and significant tachycardia in two-thirds of children tested.

Conclusion: Exertional dyspnea, cough and exercise intolerance were the most common respiratory symptoms in children with post-acute COVID-19 respiratory symptoms seen in an outpatient pulmonary clinic. Lung function, however, was mostly normal, and exertional intolerance was frequently demonstrated using the $6 \mathrm{MWT}$.

\section{Introduction}

The novel coronavirus (COVID-19) began spreading through the U.S. in early 2020, and there have been over 30 million cases in the U.S. as of June 2021, of which over 4 million have been reported in children (14.2\% of all cases). The overall rate is 5,347 cases of COVID-19 per 100,000 children in the population 1,2. Although severe illness due to COVID-19 is rare among children ${ }^{3}$, there is a lack of data on the longerterm impacts of the pandemic on children, including the long-term physical harm on the health of infected children ${ }^{4}$. Although there is little data in children, increasing reports have emerged that adult patients frequently experience long-term adverse outcomes related to COVID-19 following resolution of the acute illness $^{5-11}$. In a recent study by JK Logue et al., persistent symptoms were reported by $32.7 \%$ of adult outpatients following mild acute COVID-19 infection ${ }^{8}$. In a study of 180 mild cases of COVID-19 from the 
Faroe Islands, $53 \%$ of patients were symptomatic at mean 4-month follow up with fatigue in $30 \%$, dyspnea in $10 \%$, and chest tightness in $5 \%^{9}$. In a large longitudinal study of hospitalized patients from Wuhan, among individuals who did not require supplemental oxygen during acute COVID-19, 81\% reported residual symptoms six months after acute illness ${ }^{7}$. Previous studies have reported that even among young adults aged 18-34 years with no chronic medical conditions, approximately one in five had not returned to their usual state of health 14-21 days after acute infection ${ }^{10}$. The National Institute of Health and Care Excellence defines "long COVID" as symptoms that persistent beyond four weeks, and distinguishes between ongoing symptomatic COVID-19 as signs and symptoms of COVID-19 from four to twelve weeks and post-COVID-19 syndrome as signs and symptoms that continue for more than twelve weeks ${ }^{12}$. There is currently a paucity of literature describing such post-acute symptoms in pediatric patients with COVID-19. The aim of this study is to describe baseline health data and respiratory findings in a cohort of pediatric patients experiencing prolonged symptoms following acute COVID-19 infection.

\section{Methods}

Subjects were referred to Pulmonary Clinic at the Children's Hospital of Philadelphia (CHOP) for evaluation of persistent respiratory symptoms six weeks or more after acute COVID-19 infection between December 2020 and April 2021. Inclusion criteria included a history of SARS-CoV-2 RNA positivity or confirmed close household contact. Demographic, clinical, imaging, and functional test data were collected through retrospective chart review. Pulmonary function and six-minute walk test (6MWT) variables were referenced to predicted normal values ${ }^{13-15}$. The study was approved by the Children's Hospital of Philadelphia Institutional Review Board for exempt research.

\section{Results}

\section{Study Population}

Over a five months period, 29 pediatric subjects presented to CHOP Pulmonary Clinic for evaluation of persistent symptoms six weeks or more after COVID-19 infection (Table 1 ). Of subjects seen for COVIDrelated complaints, $14 \%$ had previously been followed in CHOP Pulmonary Clinic for other concerns prior to their acute COVID-19 infection. The mean age at presentation was 13.1 years (range: 4-19 years). Females $(58.6 \%)$ and non-whites (55.25\%) made up the majority of the patients seen. Many subjects were overweight or obese with a BMI above the 85th percentile (62.1\%). Baseline atopy, defined as presence of asthma, eczema and/or allergic rhinitis, was present in $65.5 \%$ of subjects. Of the eleven subjects with asthma, $55 \%$ had mild asthma, $36 \%$ had moderate asthma, and $9 \%$ had severe asthma. Eight subjects with asthma were previously prescribed a daily inhaled corticosteroid. Most subjects (96.6\%) reported engagement in regular physical activity prior to acute COVID-19 infection.

\section{Acute COVID Illness Characteristics}

All subjects had positive SARS-CoV-2 PCR testing or confirmed close household contacts with positive SARS-CoV-2 testing at the time of initial illness. The most common reported acute COVID-19 symptoms were fever $(69 \%)$, cough $(55.2 \%)$, dyspnea (48.3\%), ageusia/anosmia (41.4\%) and myalgia (37.9\%) (Table 2 ). Only four patients (13.8\%) required hospitalization during initial illness. All four were treated with systemic steroids and one received remdesivir. No patient required intubation. One additional patient was hospitalized six weeks after acute symptom onset owing to multisystem inflammatory syndrome in children (MIS-C). Chest radiograph opacities were present in 3 of 13 patients (23\%) who had imaging performed at the time of acute illness.

\section{Post-COVID Characteristics}

Subjects presented to clinic a mean of 3.2 months after a SARS-CoV-2 positive PCR or confirmed close contact (range: 1.3 to 6.7 months) (Table 3 ). Persistent dyspnea and/or exertional dyspnea were present in nearly all $(96.6 \%)$ of the patients at the time of clinic presentation. Other commonly reported chronic symptoms were cough $(51.7 \%)$ and exercise intolerance $(48.3 \%)$. Fatigue was reported in four subjects $(13.7 \%)$. One subject had an ongoing supplemental oxygen requirement. Oxyhemoglobin saturations ranged 
from $97 \%$ to $100 \%$. Lung auscultation findings were normal in 27 subjects. Two subjects (6.9\%) had abnormal auscultatory findings including decreased breath sounds and intermittent wheezing. Spirometry was performed in 28 patients. Mean percent predicted spirometry results included an $\mathrm{FEV}_{1}$ of $107 \%$, an $\mathrm{FVC}$ of $110 \%$, an $\mathrm{FEV}_{1} / \mathrm{FVC}$ of $86 \%$, and an $\mathrm{FEF} 25-75 \%$ of $100 \%$. Improvement following bronchodilator administration was observed in $47.6 \%$ of the 21 subjects who underwent post-bronchodilator testing. Broncho-responsiveness occurred in ten patients, five of whom have known underlying asthma and five of whom do not. Plethysmography and diffusion capacity testing were performed in 14 subjects. Average percent predicted plethysmography results include a TLC of $108 \%$, a VC of $120 \%$, an FRC of $99 \%$, and a RV of $87 \%$. An elevated RV/TLC above $30 \%$ was observed in six patients ranging from $32 \%$ to $89 \%$, suggestive of air trapping. Average percent predicted DLCO was $95 \%$ and DLCO/VA was $97 \%$. Exercise intolerance on a six-minute walk test was observed in five out of nine patients $(66.7 \%)$. Notably, these patients were found to have significant tachycardia for age (HR range: 120-213 bpm). Of the eight patients who had chest radiographs performed at the time of follow-up, only one was abnormal (12.5\%). All of the 15 patients who had follow-up cardiac evaluations had normal EKG and/or echocardiogram (Table 3 ).

\section{Discussion}

This is a descriptive study of patients referred to pediatric pulmonary for Post-Acute Sequelae of SARSCoV-2 infection (PASC), as defined by the National Institute of Health and Care Excellence guidelines ${ }^{12}$. Most patients seen for protracted respiratory symptoms following COVID-19 infection were not hospitalized and had mild acute-COVID-19 symptomatology. Protracted respiratory symptoms post-COVID-19 occurred in children as young as 4-years-old. In comparable studies that included adults who did not require hospitalization during initial COVID-19 illness, the most common complaints were dyspnea and fatigue ${ }^{5,8}$. In contrast, in our pediatric study population, fatigue was only reported by four patients (13.8\%). Similar to our study in which we found that exertional dyspnea was presented in almost all patients, Townsend et al. reported that $62 \%$ of adult survivors of COVID-19 reported persistent dyspnea and exertional limitation 75 days after their acute illness. Interestingly, persistent dyspnea and exertional limitation were not associated with initial disease severity in their study ${ }^{16}$.

In our study, although patients reported persistent exertional dyspnea, cough and exercise intolerance, lung function was normal in most children. Although objective measures of pulmonary function were generally normal, including pulse oximetry, imaging and pulmonary function testing, the six-minute walk test revealed exercise intolerance and significant tachycardia in two-thirds of children tested. This finding suggests that the six-minute walk test may be a low-cost and simple way to evaluate children after COVID-19 and provide a quantitative result that can be trended over time to assess improvement or progression of disability.

We found an increased prevalence of atopy and obesity in our study population that presented with prolonged post-COVID-19 respiratory symptoms. According to the CDC, the most recent national asthma data from 2019 suggests an asthma prevalence of $7 \%$ in children under 18 years ${ }^{17}$. The asthma prevalence in our study population was $37.9 \%$, which is nearly five times higher than the general population, and two-thirds were atopic. This may suggest that asthma and atopy are risk factors for developing long COVID respiratory symptoms in children and will require larger studies to confirm this association. Obesity, defined as a BMI above the $95^{\text {th }}$ percentile, may also be linked to persistent symptoms after COVID-19. Our study population has a higher rate of child obesity of $37.9 \%$ compared to the local population rate of $20.3 \%{ }^{18}$; however, this again may reflect study sample bias.

More studies are needed regarding effective management of patients with post-COVID syndrome. Existing guidelines and rehabilitation programs are directed at adult survivors of COVID-19 and recommend multidisciplinary treatment approaches including pulmonary rehabilitation and physical therapy ${ }^{19}$. There are limited studies that report improvement in persistent symptoms following vaccination ${ }^{20}$, but so far there are no studies looking at the impact of vaccination in pediatric patients. Pacing and gradual return to exercise may lessen exertional symptoms ${ }^{19}$. Additionally, the high prevalence of asthma and improvement following bronchodilator administration observed in $47.6 \%$ of patients suggests that there may be a role for bronchodilators and inhaled corticosteroids in some pediatric patients. 
Our population was recruited as a convenience sample and represents a limitation to our study. Patients in this study were seen in a pediatric pulmonary clinic and thus may be more motivated to seek out specialty care or have fewer barriers to accessing care. It is likely that our sample represents a small fraction of total cases of protracted COVID-19 symptoms in children within our catchment area, with the majority of cases being seen by primary care physicians. The youngest subject in our population was four years old, which may reflect the youngest age for subjects to actively report symptoms, thus we cannot comment on whether these protracted symptoms could be observed in even younger patients.

The burden of caring for COVID-19 survivors is expected to be tremendous, and future medical and social interventions must consider the late sequelae of SARS-CoV-2 infection. Even though children experience less severe COVID-19 infections than adults, our study shows that children can have significant long-term respiratory symptoms which can impact quality of life in these children. Our study indicates that ongoing respiratory morbidity can persist even in children with mild acute-COVID-19 infection history and when impairments are not clearly identified by available objective testing. This suggests that there will be an increasing need to follow these children longitudinally to determine the long-term consequences of COVID19 infection on respiratory health, as this information will have important implications for public health surveillance, health resource allocation, clinical research, and future treatments.

\section{Acknowledgements}

The authors wish to thank the patients and their families who participated in this study.

\section{References}

1. COVID Data Tracker. CDC; 2021. https://covid.cdc.gov/covid-data-tracker/\#datatracker-home.

2. American Academy of Pediatrics CsHA. Children and COVID-19: State-Level Data Report. 2021. Accessed June 23, 2021.

3. Zimmermann P, Curtis N. Coronavirus Infections in Children Including COVID-19: An Overview of the Epidemiology, Clinical Features, Diagnosis, Treatment and Prevention Options in Children. The Pediatric infectious disease journal. 2020;39(5):355-368.

4. Yelin D, Wirtheim E, Vetter P, et al. Long-term consequences of COVID-19: research needs. The Lancet Infectious Diseases.2020;20(10):1115-1117.

5. Carfi A, Bernabei R, Landi F. Persistent Symptoms in Patients After Acute COVID-19. Jama. 2020;324(6):603-605.

6. Abdallah SJ VN, Corrales-Medina VF, McGuinty M, Pratt A, Chopra A, Law A, Garuba HA, Thavorn K, Reid RER, Lavoie KL, Crawley A, Chirinos JA, Cowan J. Symptoms, Pulmonary Function and Functional Capacity Four Months after COVID-19. Annals of the American Thoracic Society.2021.

7. Huang C, Huang L, Wang Y, et al. 6-month consequences of COVID-19 in patients discharged from hospital: a cohort study. The Lancet.2021;397(10270):220-232.

8. Logue JK, Franko NM, McCulloch DJ, et al. Sequelae in Adults at 6 Months After COVID-19 Infection. JAMA Network Open.2021;4(2):e210830-e210830.

9. Petersen MS, Kristiansen MF, Hanusson KD, et al. Long COVID in the Faroe Islands - a longitudinal study among non-hospitalized patients. Clinical infectious diseases : an official publication of the Infectious Diseases Society of America. 2020.

10. Tenforde MW KS, Lindsell CJ, et al. Symptom Duration and Risk Factors for Delayed Return to Usual Health Among Outpatients with COVID-19 in a Multistate Health Care Systems Network - United States, March-June 2020. MMWR and Morbidity and Mortality Weekly Report.2020;69:993-998.

11. Morin L, Savale L, Pham T, et al. Four-Month Clinical Status of a Cohort of Patients After Hospitalization for COVID-19. Jama.2021;325(15):1525-1534. 
12. Excellence NIfHaC. COVID-19 rapid guideline: managing the long-term effects of COVID-19. London: National Institute for Health and Care Excellence (UK); December 18, 20202020.

13. Stanojevic S, Wade A, Stocks J, et al. Reference ranges for spirometry across all ages: a new approach. Am J Respir Crit Care Med. 2008;177(3):253-260.

14. Miller MR, Hankinson J, Brusasco V, et al. Standardisation of spirometry. The European respiratory journal. 2005;26(2):319-338.

15. ATS Statement: Guidelines for the Six-Minute Walk Test.American Journal of Respiratory and Critical Care Medicine.2002;166(1):111-117.

16. Townsend L, Dowds J, O'Brien K, et al. Persistent Poor Health after COVID-19 Is Not Associated with Respiratory Complications or Initial Disease Severity. Ann Am Thorac Soc. 2021;18(6):997-1003.

17. National Current Asthma Prevalence. CDC; 2019. https://www.cdc.gov/asthma/most_recent_national_asthma_data.htm.

18. Robbins JM, Mallya G, Wagner A, Buehler JW. Prevalence, Disparities, and Trends in Obesity and Severe Obesity Among Students in the School District of Philadelphia, Pennsylvania, 2006-2013. Preventing chronic disease. 2015;12:E134.

19. Salman D, Vishnubala D, Le Feuvre P, et al. Returning to physical activity after covid-19. BMJ. 2021;372:m4721.

20. Arnold D, Milne A, Samms E, Stadon L, Maskell N, Hamilton F. Are vaccines safe in patients with Long COVID? A prospective observational study. medRxiv. 2021:2021.2003.2011.21253225.

\section{Hosted file}

Long COVID in Children Tables.docx available at https://authorea.com/users/423023/articles/ 528597-protracted-respiratory-findings-in-children-post-covid-19-infection 